\title{
LAS REPERCUSIONES ESPACIALES DE LAS POLÍTICAS AMBIENTALES EN EL VÉNETO (ITALIA)*
}

\author{
María Hernández Hernández
}

\section{RESUMEN}

La política agraria emanada de la reforma McSharry aboga por la adopción de políticas que favorezcan la utilización de prácticas más compatibles con el medio físico y el sostenimiento de la población en las zonas rurales mediante la revalorización de los recursos y los espacios naturales. Esta iniciativa propugna, asimismo, la sustitución de la política de sostenimiento de la producción que ha generado cuantiosos excedentes por la de mantenimiento de las rentas. Estos nuevos postulados implican una serie de medidas de acompañamiento (reforestación de antiguas tierras agrícolas o la adopción de técnicas compatibles con el medio ambiente) para evitar una reducción drástica de las rentas de los agricultores. Estas disposiciones presentan en el Véneto unas repercusiones puntuales como consecuencia de las incompatiblidades y solapamientos que se vislumbran entre diversos reglamentos, la lentitud en la adopción de las disposiciones comunitarias o el farragoso procedimiento para su tramitación.

Palabras clave: PAC, Reforma McSharry, reforestación tierras agrícolas, Véneto, agricultura ecológica, extensificación.

\section{ABSTRAC}

The rural policy come from McSharry's reform supports the approval of programmes that promote the adoption of methods more compatible with the environment and holding the population in rural areas by the reassessment of resources and natural spaces. This measure defends, in like manner, the replacement of the policy of holding the production for the incomes policy. The former has produced considerable surplus. These new propositions involve a series of supplemental measures (reafforestation of agricultural lands or the adoption of techniques compatible with the environment) to avoid a spectacular reduction of farmers' incomes. These measures show in the Venetian region local effects. These are consequence of incompatibilities and overlapping

* Investigación realizada gracias a una beca postdoctoral del Ministerio de Asuntos Exteriores Italiano y que se desarrolló del 01-01-1998 al 31-08-98 en el departamento de Geografía de la Università degli Studi di Padova, bajo la dirección del catedrático Dario Croce. 
between laws, slowness in the passing of EU laws or the complex procedure for their negotiation.

Key words: PAC, McSharry's reform, reafforestation agricultural land, venetian region, biologic agriculture.

\section{Introducción}

En 1957 al firmarse el Tratado de Roma, los objetivos de la política agraria comunitaria se cifraban en la equiparación de las rentas de los agricultores con la del resto de la población, la incentivación de la producción para generar alimentos a precios bajos y la mejora de la productividad para asegurar el abastecimiento de alimentos. Para lograr estos fines se estableció un sistema de apoyo garantizado con la creación del Fondo de Orientación y Garantía Agrícola y fundamentado en los principios de unidad de mercado, la preferencia comunitaria y la solidaridad financiera (Lázaro Arujo, L. y Molina Ibáñez, M.; 1986).

La política de apoyo descrita permitió un rapidísimo crecimiento de la producción y de los rendimientos hasta dar lugar a la aparición de cuantiosos excedentes. No son, sin embargo, éstos la única consecuencia negativa de las iniciativas comunitarias. Debemos citar, por un lado, la degradación ambiental, evidenciada por el Club de Roma a principios de los años setenta y, por otro, la marginalización creciente de ciertos espacios (áreas montañosas). Este último proceso fue consecuencia de los propios elementos definidores (orografía intrincada, elevadas pendientes, etc.) que dificultaban su adaptación a los nuevos postulados económicos (intensificación de la explotación, obtención de elevados rendimientos, etc.) y tecnológicos (mecanización) que se difunden a partir de los años cincuenta. Aspectos que unidos a la existencia de áreas que atraen a la población gracias a las mejores salarios ofertados generarán un intenso éxodo rural y la desorganización paulatina de estos espacios, lo que acentuará los desequilibrios regionales.

Los rasgos citados unido al elevado porcentaje del presupuesto comunitario destinado al sector primario (en torno al 80\%) suscitaron un debate en el seno de la comisión sobre la necesidad de una reforma en profundidad de la política agraria común. No obstante, esta determinación no es novedosa sino que se remonta a finales de los años sesenta con la elaboración del informe Mansholt $(1968)^{1}$, también conocido como Programa Agricultura 80 , donde por primera vez se hace referencia a la aparición de excedentes fruto de unas estructuras de producción inapropiadas. Éstos, unidos a la aparición de grupos en países como Holanda y Alemania que demandaban la adopción de técnicas más compatibles con el medio ambiente, justifican la aprobación en 1970 de un reglamento que regulaba la utilización de los productos fitosanitarios ${ }^{2}$.

1 Esta reforma aboga por una transformación progresiva de las estructuras agrarias con el objetivo de construir unidades de producción más modernas y racionalizadas.

2 La regulación de los productos fitosanitarios y la adopción de disposiciones que incentivasen la adopción de técnicas compatibles con el medio ambiente, entre las que destaca la agricultura ecológica, fueron contempladas por primera vez en 1970 con la aprobación del reglamento 729/70. Disposición que, desde el punto de vista de las repercusiones en el medio rural, tuvo un carácter meramente testimonial; siendo sustituido por el 2048/88. 
A pesar de estas iniciativas, los principios productivistas no comenzarán a ser cuestionados de manera irrebatible hasta mediados de la década de los ochenta. Estas críticas se plasmarán en informes como Reflexiones sobre la Política Agraria Común, Perspectivas sobre la Política Agraria comunitaria, El futuro del mundo rural y Agricultura y medio ambiente. En los documentos citados se aboga por la adopción de iniciativas que favorezcan, por un lado, el sostenimiento de la población en las zonas rurales, concediendo un mayor protagonismo a los recursos locales a los que se considera elementos prioritarios para su desarrollo y, por otro, la adopción de prácticas menos «duras» hacia el entorno (extensificación, reducción en el uso de productos fitosanitarios, etc.). No obstante, la valoración de los resultados obtenidos en este decenio fue más bien mediocre ya que las medidas adoptadas pretendían más que evitar los procesos de degradación ambiental, reconducirlos a unos porcentajes aceptables (Francescheti, G. y Tempesta, T.; 1993). Afirmación corroborada por el tipo de medidas adoptadas: hasta finales de los ochenta se circunscribieron a la imposición de cuotas y a la reducción de la superficie cultivada ya que las principales ayudas se orientaron a la retirada de tierras de la producción, tal y como sucedió con los cereales ${ }^{3}$.

Las directrices teóricas de esos informes se mantendrán en documentos posteriores: en un primer momento en el Acta Única (1987) y, posteriormente, en la reforma de 1992 (reforma McSharry), que consideraba prioritario la reducción de la superproducción, favorecer el desarrollo de actividades económicas alternativas y la protección del medio ambiente. Abogando, asimismo, por el abandono del sistema de protección vigente fundamentado en las ayudas a la producción al que se acusa de generar excedentes, por el sostenimiento directo de las rentas. Aprobándose una serie de disposiciones conocidas como «medidas de acompañamiento» cuyo objetivo último es compensar la pérdida de rentas generadas por el cambio de política.

Éstas comprenden dos reglamentos el 2078 y el 2080/92. El primero regula la adopción de métodos de producción agrícola compatibles con el medio ambiente. Englobando actuaciones como la adopción de sistemas de producción con bajo impacto ambiental, la extensificación de terrazgos productivos, la recuperación de áreas agrícolas y forestales marginales, la restauración de paisajes agrarios tradicionales o la potenciación de actividades recreativas en las áreas rurales. Incorporado también disposiciones aprobadas con anterioridad como la 2091/92, que normaliza la práctica de la agricultura ecológica. El segundo regula la reforestación de antiguas tierras, instituyendo un nuevo régimen de ayudas para la reforestación de tierras agrícolas, dado la exigua aceptación de disposiciones anteriores. A las disposiciones citadas se suman aquéllas que contemplan la temática ambiental desde una óptica sectorial; actuaciones que habían sido dominantes antes de la aprobación de la reforma de 1992. Entre éstas merecen citarse los reglamentos 1765 y 1766/92 relativos a la organización común del mercado de los cereales o los propios reglamentos estructurales (797/85 y 2328/91), que consideraban la reforestación como una de sus líneas de actuación.

3 El reglamento comunitario 1049/88 regulaba la retirada obligatoria de superficies cerealícolas. El 1272 y 1273/88 contenían los aprovechamientos a introducir en estos terrazgos (barbecho con posible rotación, transformación en pastos para la ganadería extensiva, cultivo de legumbres o su utilización para fines no agrarios). Adaptándose a la legislación italiana mediante los decretos 34/89, 35/90 y 63/91, respectivamente. Estableciendo una serie de ayudas directas que se cifran en 600 ecus/ha para explotaciones en la llanura padano-véneta, 440 para las de otras llanuras y 380 para las explotaciones en áreas colinares marginales y áreas montañosas (lámina 1). 
Estos planteamientos generales, no obstante, presentan un tratamiento diferenciado según las características del área agrícola afectada (zonas marginales y áreas de elevada vocación agrícola). En estas últimas, la comisión aboga por la tutela de los terrazgos más aptos vinculados al ejercicio de una actividad agraria intensiva en donde se incentiva la adopción de tecnologías compatibles, la racionalización en el uso de los recursos renovables, la realización de estudios de impacto ambiental en aquellas actividades que generen significativos procesos de degradación y la adopción de una serie de políticas de denominación de origen cuyo objetivo último sería reconvertir una producción de cantidad en una de calidad.

Las actuaciones dependientes de la política agraria son limitadas en estos terrazgos ya que sus elementos definidores permiten la obtención de rentas suficientes gracias a su elevada competitividad. En cambio, éstas se intensifican en las zonas marginales. Orientándose a la incentivación directa de los ingresos de los agricultores cuando lleven a cabo actividades de tutela ambiental mediante la reconversión agroforestal o la adopción de técnicas compatibles con el medio ambiente. Otras medidas se encaminan a la difusión de formas de desarrollo local integrado mediante la valorización de los productos agrícolas y artesanales típicos de la zona y actividades vinculadas a la práctica del turismo de interior. Propuestas que, grosso modo, se resumen en dos afirmaciones como es la necesidad de mantener un número suficiente de agricultores y reconocer el hecho de que éstos desempeñan dos funciones de forma simultánea: actividad productiva y, de protección del medio ambiente.

\section{Repercusiones socio-espaciales de las medidas de acompañamiento de la reforma Mcsharry en el Véneto}

La política agraria comunitaria, cuyos elementos definidores se especificaron en el epígrafe anterior, ha sido incorporada a la legislación véneta tras la aprobación por el gobierno regional del Piano Territoriale Regionale di Coordinamento (P.T.R.C), en el que se inserta el «Programma Regionale di Sviluppo Agricolo e Forestale 1990-94» y su continuación para el periodo 1996-2000, en el que se definen las prioridades del sector agrícola véneto y donde se concede una gran relevancia a la temática agroambiental.

\subsection{La reforestación de antiguas tierras agrícolas}

Los bosques ocupan en el Véneto una superficie en torno a 271.646 has es decir, el $19,5 \%$ de la superficie total. No obstante, estas cifras enmascaran una notable heterogeneidad regional fruto de la variedad orográfica que caracteriza a este ámbito: en las áreas montanas y concretamente, la provincia de Belluno, ésta alcanzaría el $44 \%$ frente al 2\% de las provincias de Rovigo y Padua, donde el elemento dominante son las amplias llanuras fruto de la bonificación (cuadro 1). Superficie en progresivo incremento no sólo por las iniciativas estatales $^{4}$, sino, sobre todo, por el abandono de terrazgos agrícolas en áreas marginales, el control de las talas, el abandono de prados y las nuevas plantaciones debido a las ayudas comunitarias (cuadro 2).

4 En el periodo comprendido entre 1992-96 se aprobaron 139 planes de reforestación que afectaban a una superficie total de 226.000 hectáreas, de las cuales 105.000 correspondían a prados, pastos y áreas improductivas, generalmente de propiedad pública. Concentrándose en un $70 \%$ en las áreas montañosas y el porcentaje restante en el área colinar. 
Cuadro 1

SUPERFICIE FORESTAL EN LA REGIÓN VÉNETO

\begin{tabular}{|l|r|r|r|r|r|c|}
\hline & \multicolumn{1}{|c|}{ SAU } & \multicolumn{3}{|c|}{ Sup arbolada } & otras sup & sup total \\
\cline { 2 - 5 } & Sup & Chopos & Bosques & $\%$ & & \\
\hline Belluno & 108366,57 & --- & 127745,01 & 43,61 & 12345,67 & 248457,25 \\
Padua & 140506,01 & 403,04 & 3387,03 & 2,31 & 19287,95 & 163584,03 \\
Rovigo & 119541,36 & 1384,95 & 28,75 & 1,03 & 15879,67 & 136834,73 \\
Treviso & 142641,26 & 627,25 & 19025,68 & 10,65 & 22187,59 & 184481,78 \\
Venecia & 122940,86 & 647,82 & 9736,21 & 6,67 & 22156,95 & 155491,84 \\
Verona & 180962,67 & 738,24 & 18613,80 & 8,82 & 18897,95 & 219212,66 \\
Vicenza & 119486,93 & 18,59 & 57465,33 & 29,19 & 19838,63 & 196809,48 \\
\hline
\end{tabular}

Fuente: $4^{\circ}$ Censimento generale dell'agricoltura, Provincias de Rovigo, Verona, Venecia, Treviso y Padua. Elaboración propia.

Cuadro 2

EVOLUCIÓN DE LA SUPERFICIE FORESTAL EN EL VÉNETO (1971-1991)

\begin{tabular}{|c|c|c|c|c|c|c|c|}
\hline & & \multicolumn{3}{|c|}{1971} & \multicolumn{3}{|c|}{1991} \\
\hline & & $\mathbf{N}^{0}$ & Sup & $\%$ & $n^{0}$ & Sup & $\%$ \\
\hline Belluno & $\begin{array}{l}\text { Chopos } \\
\text { Bosque }\end{array}$ & 16492 & 129456 & 64,49 & 13996 & 127746 & 63,30 \\
\hline Padua & $\begin{array}{l}\text { Chopos } \\
\text { Bosque }\end{array}$ & $\begin{array}{r}19 \\
2190\end{array}$ & $\begin{array}{r}22,55 \\
3881,33\end{array}$ & $\begin{array}{l}0,01 \\
2,24\end{array}$ & $\begin{array}{r}305 \\
1742\end{array}$ & $\begin{array}{r}403,04 \\
3387,03\end{array}$ & $\begin{array}{l}0,20 \\
2,18\end{array}$ \\
\hline Rovigo & $\begin{array}{l}\text { Chopos } \\
\text { Bosques }\end{array}$ & $\begin{array}{r}29 \\
180\end{array}$ & $\begin{array}{r}99,03 \\
318,60\end{array}$ & $\begin{array}{l}0,15 \\
0,23\end{array}$ & $\begin{array}{r}248 \\
30\end{array}$ & $\begin{array}{r}1384,95 \\
28,75\end{array}$ & $\begin{array}{l}1,23 \\
0,01\end{array}$ \\
\hline Treviso & $\begin{array}{l}\text { Chopos } \\
\text { Bosques }\end{array}$ & $\begin{array}{r}67 \\
12686\end{array}$ & $\begin{array}{r}68,06 \\
22579,97\end{array}$ & $\begin{array}{r}0,01 \\
11,01\end{array}$ & $\begin{array}{r}482 \\
11318\end{array}$ & $\begin{array}{r}647,25 \\
19025,68\end{array}$ & $\begin{array}{r}0,31 \\
22,10\end{array}$ \\
\hline Venecia & $\begin{array}{l}\text { chopos } \\
\text { bosques }\end{array}$ & $\begin{array}{r}88 \\
364\end{array}$ & $\begin{array}{l}234,57 \\
787,21\end{array}$ & $\begin{array}{l}0,23 \\
0,52\end{array}$ & $\begin{array}{l}227 \\
347\end{array}$ & $\begin{array}{r}647,82 \\
9746,21\end{array}$ & $\begin{array}{l}0,43 \\
6,37\end{array}$ \\
\hline Verona & $\begin{array}{l}\text { chopos } \\
\text { bosques }\end{array}$ & $\begin{array}{r}30 \\
7897\end{array}$ & $\begin{array}{r}149,19 \\
25029,30\end{array}$ & $\begin{array}{r}0,12 \\
10,14\end{array}$ & $\begin{array}{r}156 \\
7111\end{array}$ & $\begin{array}{r}738,24 \\
18613,80\end{array}$ & $\begin{array}{l}0,32 \\
8,58\end{array}$ \\
\hline Vicenza & $\begin{array}{l}\text { chopos } \\
\text { bosques }\end{array}$ & $\begin{array}{r}17 \\
18302\end{array}$ & $\begin{array}{r}28,35 \\
58033,16\end{array}$ & $\begin{array}{r}0,02 \\
25,94\end{array}$ & $\begin{array}{r}19 \\
17776\end{array}$ & $\begin{array}{r}18,59 \\
57465,33\end{array}$ & $\begin{array}{r}0,01 \\
29,19\end{array}$ \\
\hline
\end{tabular}

FuEnTE: $4^{\circ}$ Censimento generale dell'agricoltura, Provincias de Rovigo, Verona, Venecia, Treviso y Padua. Elaboración propia. 


\subsubsection{Reglamentos sectoriales}

Hasta finales de los ochenta, las políticas comunitarias consideraban el medio ambiente desde un punto de vista tangencial, tal y como sucedía con los reglamentos estructurales (795/85 y 2328/92) y los que regulaban la OCM de los cereales (1765 y 1766/92). Los primeros incluían la reforestación en una de sus líneas de actuación, sin embargo su aceptación fue muy escasa (menos de un $1 \%$ de las peticiones totales). Esta baja aceptación es extensible al resto de las líneas de actuación (incorporación de jóvenes, modernización de las estructuras, etc.) dada la escasa relevancia que el sector agrícola tiene en el Véneto: genera únicamente el 4,16\% del PIB regional. Este valor la convierte en una actividad escasamente dinámica; rasgo acentuado por una serie de aspectos como el reducido número de activos $^{5}$, su elevado envejecimiento ${ }^{6}$ y el predominio de agricultores a tiempo parcial ${ }^{7}$ (Hernández Hernández, M.; 1999). Estos aspectos determinan que las peticiones procedan mayoritariamente de los agricultores profesionales, una minoría, que solicitan ayudas para mejorar unas explotaciones generalmente intensivas (aprovechamientos hortícolas o leñosos mediante la introducción de sistemas de producción bajo plástico, la mejora varietal, introducción de nuevos cultivos, mejora de las explotaciones, compra de maquinaria, etc.) y donde la introducción de aprovechamientos forestales es desechada, al considerarla como una «subvención» y no una verdadera opción que mejore su explotación y consiguientemente sus rentas.

Una aceptación similar presentan los reglamentos que regulan la organización común de mercado (OCM) de los cereales. Aprovechamientos que son dominantes (cuadro 3) en la denominada llanura padana, dados los condicionamientos físicos (antiguas áreas bonificadas) que determinan la existencia de unos parcelarios amplios fácilmente mecanizables. Además, dados las menores atenciones que requieren se adaptan perfectamente a la práctica de una agricultura a tiempo parcial; contando a su vez con un importante apoyo como son las subvenciones comunitarias. La amplia superficie ocupada por los cereales, unas 606.929 has. (censo agrario de 1991) se traduce en un porcentaje de tierra dejada en reposo (set asside), que se sitúa alrededor de $15 \%$ del terrazgo cultivado. Normativa de obligado cumplimento para aquellos propietarios que superen una producción de 92 toneladas; obteniéndose ésta en explotaciones con una extensión de unas 11 has. Regulando éstos, asimismo, los usos a introducir en ellos (barbecho, reforestación, leguminosas, pastos para ganadería extensiva).

5 En 1951, el sector primario ocupaba al 43\% de la población en edad de trabajar frente al 5,82\% de 1991. Descenso que debemos relacionar con la difusa industrialización que caracteriza a esta región y que se tradujo en una primera etapa en el incremento de los activos secundarios y, posteriormente, una terciarización de la sociedad, ocupando estos sectores, según el último censo, al 43,9 y 50,3\% de los asalariados, respectivamente.

6 Los activos con más de 55 años alcanzan porcentajes que oscilan, según el Censo de Población de 1991, entre el 70,83\% de Rovigo y el 56,21\% de Belluno.

7 Los porcentajes de los activos a tiempo parcial oscilan entre el 70,83\% de Rovigo y el 56,21\% de Belluno. 
Cuadro 3

EVOLUCIÓN DE LOS APROVECHAMIENTOS AGRARIOS: 1971-1991

\begin{tabular}{|c|c|c|c|c|c|c|c|c|c|}
\hline & \multirow[t]{3}{*}{ sup.total(ha) } & \multirow{2}{*}{\multicolumn{2}{|c|}{$\operatorname{SAU}(\%)$}} & \multicolumn{4}{|c|}{ Herbáceos $(\%)^{*}$} & \multicolumn{2}{|c|}{ Leñosos $(\%)^{*}$} \\
\hline & & & & \multirow[b]{2}{*}{1971} & \multirow[b]{2}{*}{1991} & \multicolumn{2}{|c|}{ Hortalizas $^{\mathrm{a}}$} & \multirow[b]{2}{*}{1971} & \multirow[b]{2}{*}{1991} \\
\hline & & 1971 & 1991 & & & 1971 & 1991 & & \\
\hline Padua & 163584,03 & 88,22 & 85,89 & 79,48 & 84,52 & 1,06 & 2,59 & 14,01 & 10,34 \\
\hline Rovigo & 136834,73 & 87,98 & 87,36 & 89,53 & 95,48 & 3,81 & 4,79 & 7,91 & 4,31 \\
\hline Treviso & 184480,78 & 79,76 & 77,32 & 57,60 & 63,51 & 0,63 & 0,92 & 21,19 & 19,73 \\
\hline Venecia & 155491,84 & 93,78 & 79,06 & 82,77 & 90,26 & 2,10 & 2,95 & 14,82 & 8,27 \\
\hline Verona & 219212,66 & 90,34 & 82,55 & 53,02 & 54,39 & 4,62 & 4,55 & 24,44 & 26,04 \\
\hline Vicenza & 196809,48 & 73,02 & 60,71 & 45,06 & 46,15 & 0,91 & 1,32 & 9,71 & 9,23 \\
\hline
\end{tabular}

* Porcentajes realizados sobre la superficie agraria útil; ${ }^{\text {a: }}$ porcentajes sobre la superficie de herbáceos. Fuente: Censos Agrarios de 1971 y 1991. Elaboración propia.

El análisis de los aprovechamientos introducidos (predominio abrumador del barbecho) en estos terrazgos evidencia el mantenimiento de la estructura agraria dominante: la rotación de la superficie no cultivada permite continuar con ese sistema productivo, además de no ocasionar ningún coste adicional su ejecución. Son poco representativos los agricultores que introducen usos alternativos en la superficie dejada en reposo (alrededor de un $10 \%$, distribuida entre un $3 \%$ en reforestación y el resto dedicada al cultivo de legumbres o los pastos para la ganadería extensiva). Estos usos alternativos ven dificultados su difusión por problemas estructurales: los primeros encuentran problemas a la hora de su comercialización por lo que no es extraño que no se recolecten y sean utilizados como abono orgánico y los segundos debido a la falta de integración entre agricultura y ganadería. Esta última es prácticamente inexistente en el área donde predominan los cereales de ahí que no sea viable desde un punto de vista económico este uso (pastos para la ganadería extensiva).

\subsubsection{El reglamento 2080/92}

La escasa aceptación de estas disposiciones trató de ser paliada con la aprobación del reglamento 1096/89, en el que por primera vez la mejora o la ampliación de las superficies arboladas era el principal objetivo. No obstante, tampoco obtuvo resultados satisfactorios. Tendencia que trata de ser invertida con el 2080/92 mediante la mejora de una serie aspectos que estaban insuficientemente recogidos en disposiciones anteriores o la introducción de nuevos postulados. Entre los primeros debemos citar el incremento de la aportación pública $^{8}$ y de las subvenciones percibidas, al diferenciar entre gastos de reforestación, de mantenimiento y una prima para compensar la reducción de rentas de los agricultores (Hernández Hernández, M.; 1998).

8 Programa financiado con cargo al FEOGA-Garantía que subvenciona el 75\% de las inversiones en las regiones objetivo 1 y el $50 \%$ en las restantes. Ayudas incrementadas con respecto a iniciativas anteriores en un $25 \%$. 
Modificaciones que han sido recogidas en el Programa Regional de Desarrollo Agrícola y Forestal y en el plan sectorial para las áreas montanas (Piano per lo sviluppo socio economico e ambientale della montagna). Estas actuaciones constituyen una innovación notable con respecto a la programas anteriores donde la reforestación en áreas montanas ${ }^{9}$ era prioritaria; posición que ceden a la llanura y a las colinas donde ésta se considera un elemento capaz de reducir los problemas de degradación ambiental y social que la desorganización de los sistemas productivos ha ocasionado tal y como se observa en la Lesinia o los montes Berici.

El presupuesto disponible para el periodo 1993-97, que asciende a 22.001.063 ecus, junto a los condicionamientos físicos de la región, podrían hacer pensar en una amplia aceptación de este programa comunitario. Sin embargo, el análisis de las solicitudes presentadas evidencia un cierto fracaso (cuadro 4) pues éstas únicamente han interesado 25.048.159 euros, es decir, el $62 \%$ del coste previsto del programa. Siendo incluso más decepcionante si se considera el porcentaje de las peticiones realizadas, que se sitúa en torno al 32\% del capital total asignado a este programa, es decir, unos 9.141.287,1 ecus.

\section{Cuadro 4}

EVOLUCIÓN NÚMERO SOLICITUDES Y SUPERFICIES

\begin{tabular}{|l|rr|rr|rr|rr|}
\hline & \multicolumn{2}{|c|}{$\mathbf{1 9 9 4}$} & \multicolumn{2}{c|}{$\mathbf{1 9 9 5}$} & \multicolumn{2}{c|}{$\mathbf{1 9 9 6}$} & \multicolumn{2}{|c|}{1997} \\
\cline { 2 - 9 } & $\mathbf{n}^{\mathbf{0}}$ & sup & $\mathbf{n}^{\mathbf{0}}$ & sup & $\mathbf{n}^{\mathbf{0}}$ & sup & $\mathbf{n}^{\mathbf{0}}$ & sup \\
\hline Presentadas & 267 & 902 & 304 & 847 & 194 & 543 & 303 & 931 \\
Admitidas & 198 & 642 & 221 & 583 & 164 & 456 & 254 & 806 \\
Realizadas & 135 & 385 & 110 & 281 & 58 & 148 & 27 & 148 \\
\hline
\end{tabular}

FuENTE: Dipartimento per le Foreste e l'Economia Montana. Segretaria Regionale per le attività produttive de economiche del settore primario (Regione Veneto). Elaboración propia.

El elevado porcentaje de fondos disponibles no utilizados se vincula a cuestiones como el régimen de incompatibilidades ${ }^{10}$, las menores exigencias requeridas $\mathrm{y}$, consiguientemente, la competencia ejercida por programas como el Piano per la prevenzione e il risanamento delle acque del bacino idrografico que desagua en la laguna véneta (plan aprobado por la ley n ${ }^{\text {o }} 3286$ del 23-9-199711) y las ayudas previstas por el plan de protección de la fauna ${ }^{12}$ (Piano faunistico-venatorio, ley regional $n^{\circ} 17$ de 27 de junio de 1996).

9 El programa prohibe la creación en zonas de montaña de zonas boscosas cuyo objetivo sea el paisajístico; prevé, en cambio, ayudas para la conservación y mejora de superficies ya forestadas.

10 Es incompatible acogerse simultáneamente al programa de reforestación de tierras agrícolas (2080/92) y la adopción de técnicas compatibles con el medio ambiente (2078/92). No siendo tampoco acumulables las subvenciones percibidas procedentes del reglamento 1765/92 que regula las ayudas percibidas por las superficies con aprovechamientos cerealícolas dejadas en reposo y entre cuyos usos alternativos se encuentra la reforestación.

11 Programa que subvenciona la plantación de arboles en los canales de drenaje para evitar la aparición de procesos de desmoronamiento debido a la labor de zapa lateral de la corriente y consiguientemente el incremento del proceso natural de colmatación de la laguna.

12 Iniciativa que pretende proteger la fauna con la reducción en el uso de fertilizantes, la creación de pequeños bosquetes y el mantenimiento de la cubierta vegetal en los canales de drenaje y en los linderos de las parcelas de cultivo. 
Entre los menores requisitos requeridos una mención especial reviste la superficie mínima. Así, por ejemplo, el reglamento 2.078 concede subvenciones a aquellos agricultores que plantan hileras de árboles a lo largo de los canales de drenaje (recuperación de los paisajes tradicionales), pero sin establecer una superficie o un número mínimo de plántuals. Por el contrario, el 2.080 establece que estos «setos» (siepi) deben estar constituidos al menos por 2 hileras y una superficie mínima de una hectárea, lo que implica una parcela de dimensiones importantes.

A los rasgos citados (solapamiento, menores exigencias o la superficie mínima) se suman otras cuestiones como el quedar excluidas de las ayudas variedades de amplia difusión en el agro véneto, la exclusión de áreas de elevada vocación forestal o la escasa formación técnica de los agricultores en materia forestal que justifican esa escasa aceptación. Las superficies reforestadas con plántulas de ciclo corto (chopos para la elaboración de pasta de papel) han visto incrementada su superficie en el Véneto en la última década (cuadro 2). Sin embargo, este aumento no se vincula a esta política comunitaria sino a la iniciativa particular favorecida por los elevados rendimientos generados por este aprovechamiento forestal. La aplicación del reglamento $n^{\circ}$ 2296/92 determina que sea una minoría los cultivadores que puedan acogerse a estas ayudas. En primer lugar no perciben la prima compensatoria pero además quedan excluidos del resto de las ayudas todos aquellos agricultores no profesionales o aquellos terrazgos que dejaron de cultivarse con anterioridad al 31-12-1992.

La pérdida de prioridad de las terrazgos montañosos viene determinada por la prohibición de percibir ayudas comunitarias aquellas reforestaciones que en estas áreas persigan un objetivo meramente ambiental. Esta exclusión justifica las reducidas peticiones procedentes de estos espacios; uso que era permitido en reglamentos anteriores. Estando únicamente bonificadas las actuaciones orientadas a la mejora de los bosques existentes, la conservación de los caminos forestales, la reintroducción de frondosas o el apoyo a iniciativas privadas en campos como la planificación y la gestión de los recursos naturales. Sin embargo, estas actuaciones son meramente testimoniales (cuadro 5) dada la escasa vocación forestal de los agricultores vénetos que ven esta medida más como una «subvención más» que como una verdadera opción de desarrollo; de ahí que se muestren muy reacios a hipotecar sus tierras por un periodo mínimo de veinte años o creen puntos de agua o caminos forestales en las áreas reforestadas.

Cuadro 5

EVOLUCIÓN DE LAS PETICIONES ACOGIDAS A LA MEJORA DE LAS SUPERIFICIES FORESTADAS (1994-1997)

\begin{tabular}{|l|rr|rr|rr|rr|}
\hline & \multicolumn{2}{|c|}{1994} & \multicolumn{2}{c|}{1995} & \multicolumn{2}{c|}{1996} & \multicolumn{2}{c|}{1997} \\
\cline { 2 - 9 } & prevtas & rdas & prevtas & rdas & prevtas & rdas & prevtas & rdas \\
\hline mejora bosques* $^{*} 833,3$ & 26,05 & 833,3 & 9,20 & 833,3 & --- & 833,3 & --- \\
pistas forestales $^{1}$ & 114,6 & 1,08 & 114,6 & 0,13 & 114,6 & --- & 114,6 & --- \\
puntos de agua & 343,0 & --- & 343,0 & --- & 343,0 & --- & 243,0 & --- \\
\hline
\end{tabular}

prevtas: previstas; rdas: realizadas; * en has; ${ }^{1}$ en kilómetros; Fuente: Dipartimento per le Foreste e l'Economia Montana. Segretaria Regionale per le attività produttive ed economiche del settore primario (Regione Veneto). Elaboración propia. 
Los rasgos citados en párrafos anteriores determinan la distribución de las superficies acogidas a este reglamento (cuadro 6, lámina 1). Si éstos no se considerasen, resultaría sorprendente que fueran las provincias de Venecia y Padua, incluidas casi en su totalidad en la denominada baja llanura, las que concentrasen las mayores superficies reforestadas. Mientras que aquéllas con una aptitud potencial mayor (relieves alpinos y prealpinos y áreas colinares) tengan una presencia prácticamente testimonial. Esta distribución se corrobora además al analizar la funcionalidad de estas actuaciones: la producción de madera para trabajarla es abrumadora (70\%) frente a la conservación de la naturaleza (22\%), al quedar excluidas los sectores montañosos, o el turístico-recreativo o el multifuncional, prácticamente inexistentes (1\%). Configurándose nuevas áreas forestales entre los aprovechamientos cerealícolas (maíz preferentemente) y los cultivos industriales (soja y remolacha azucarera). Estas «islas» son el resultado de la propia estructura de la propiedad: mayoritariamente se han acogido pequeños propietarios con superficies que no superan las 3 has de media; siendo poco frecuente la existencia de actuaciones colectivas.

Cuadro 6

EVOLUCIÓN DE LAS PETICIONES (1994-1997) A ESCALA PROVINCIAL

\begin{tabular}{|c|c|c|c|c|c|c|c|c|c|c|}
\hline & \multicolumn{4}{|c|}{$\mathrm{n}^{0}$ solicitudes } & \multicolumn{6}{|c|}{ Total } \\
\hline & \multirow[t]{2}{*}{1994} & \multirow[t]{2}{*}{1995} & \multirow[t]{2}{*}{1996} & \multirow[t]{2}{*}{1997} & \multirow[t]{2}{*}{$\mathrm{n}^{0}$} & \multirow[t]{2}{*}{$\%$} & \multicolumn{4}{|c|}{ Sup } \\
\hline & & & & & & & Frond & chopo & total & $\%$ \\
\hline Belluno & 40 & 19 & 3 & 18 & 77 & 6,34 & -- & -- & --- & - \\
\hline Padua & 90 & 102 & 55 & 82 & 329 & 27,10 & 188,38 & 39,90 & 228,2 & 21,3 \\
\hline Rovigo & 29 & 20 & 43 & 18 & 110 & 9,06 & 58,12 & 78,88 & 137,0 & 12,8 \\
\hline Treviso & 71 & 88 & 47 & 97 & 303 & 24,95 & 146,59 & 85,07 & 231,6 & 21,6 \\
\hline Venecia & 40 & 54 & 24 & 73 & 191 & 15,73 & 130,27 & 196,21 & 326,4 & 30,5 \\
\hline Verona & 34 & 23 & 18 & 27 & 103 & 8,48 & 41,07 & 48,31 & 89,3 & 8,34 \\
\hline Vicenza & 28 & 38 & 13 & 19 & 98 & 8,07 & 49,59 & --- & 49,5 & 4,6 \\
\hline Total & 332 & 345 & 203 & 334 & 1214 & 100,00 & 614,02 & 446,37 & 1069,9 & 100,0 \\
\hline
\end{tabular}

FuENTE: Dipartimento per le Foreste e l'Economia Montana. Segretaria Regionale per le attività produttive ed economiche del settore primario (Regione Veneto). Elaboración propia.

\subsection{Agricultura ecológica}

El término agricultura ecológica se utiliza a menudo como sinónimo de agricultura alternativa, agricultura biológica, orgánica e incluso agricultura sostenible. Vocablos que definen un sistema de cultivo cuyo objetivo fundamental es la obtención de alimentos respetando el medio ambiente y conservando la fertilidad de la tierra, mediante la utilización óptima de los recursos y sin el empleo de productos químicos de síntesis ${ }^{13}$. Estos plantea-

13 C.R.A.E: Normas técnicas, capítulo. I-2º citado por: LÓPEZ ONTIVEROS, A. y RUIZ MOYA, J.: «Agricultura ecológica, medio ambiente y desarrollo rural», en Actas del VII Coloquio de Geografía Rural, Córdoba, 1994, pág. 208. 

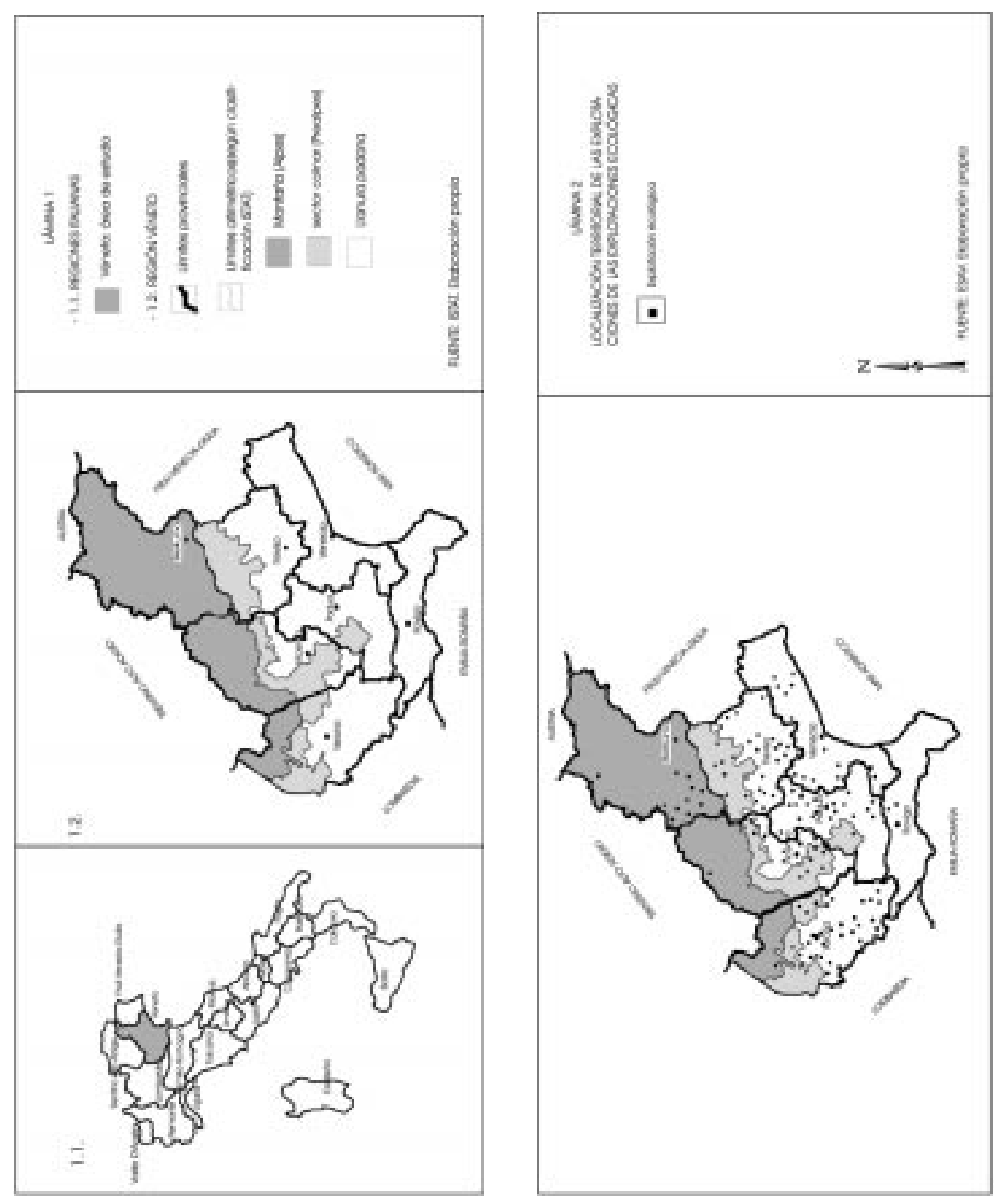
mientos se recogen en el reglamento 2092/91, que regula los principios para la producción ecológica, además de instituir un régimen de ayudas para compensar la disminución de rentas de los agricultores que se acogen a este modelo de producción ${ }^{14}$. Siendo incorporados a la legislación regional mediante la ley no 24 de 6 de abril de 1990 (Piano agricola-ambientale e per la lotta fitopatologica).

Cuadro 7

EVOLUCIÓN SUPERFICIES DEDICADAS A LA AGRICULTURA ECOLÓGICA

\begin{tabular}{|l|rr|rr|rr|rr|}
\hline & \multicolumn{2}{|c|}{$\mathbf{1 9 8 0}$} & \multicolumn{2}{|c|}{$\mathbf{1 9 9 0}$} & \multicolumn{2}{|c|}{$\mathbf{1 9 9 6}$} & \multicolumn{2}{|c|}{$\mathbf{1 9 9 8}^{*}$} \\
\cline { 2 - 9 } & $\mathbf{n}^{\mathbf{0}}$ & sup & $\mathbf{n}^{\mathbf{0}}$ & sup & $\mathbf{n}^{\mathbf{0}}$ & sup & $\mathbf{n}^{\mathbf{0}}$ & sup \\
\hline Véneto (total & 18 & 238,75 & 119 & 596,76 & 654 & 4571,77 & 759 & 5305,76 \\
Belluno & 2 & 14,50 & 9 & 42,93 & 43 & 751,02 & 54 & 821,02 \\
Padova & 2 & 1,20 & 21 & 67,46 & 84 & 319,36 & 102 & 402,12 \\
Rovigo & -- & -- & 2 & 14,93 & 9 & 103,00 & 18 & 195,00 \\
Treviso & 8 & 41,90 & 27 & 121,83 & 167 & 788,91 & 187 & 902,55 \\
Venecia & 1 & 158,00 & 12 & 112,26 & 67 & 597,27 & 78 & 703,25 \\
Verona & 4 & 22,85 & 30 & 172,03 & 208 & 1556,43 & 229 & 1775,22 \\
Vicenza & 1 & 0,30 & 18 & 65,32 & 76 & 455,78 & 91 & 506,30 \\
\hline Clasificación & & & & & & & & \\
altimétrica ${ }^{1}$ & & & & & & & & \\
\hline Montaña & 2 & 14,50 & 9 & 42,93 & 43 & 751,02 & 54 & 821,02 \\
Colina & 7 & 38,25 & 23 & 123,81 & 158 & 975,25 & 187 & 1225,75 \\
Llanura & 9 & 186,00 & 87 & 430,00 & 453 & 2845,50 & 518 & 3258,99 \\
\hline
\end{tabular}

Datos a 30 de mayo de 1998. 'clasificación adaptada a la terminología empleada por el ISTAT. Fuente: Ente Sviluppo Agrario del Veneto. Elaboración propia.

En el Véneto, las primeras explotaciones que adoptan técnicas ecológicas se remontan a finales de la década de los setenta: en 1980 se estimaba que unas 18 unidades productivas cultivaban con técnicas no convencionales. Cifras que van a experimentar un notable crecimiento durante la década de los ochenta y los noventa: en 1990, las explotaciones ascendían a 119 y la superficie se situaba en torno a las 600 hectáreas (Boatto, V. et al.: 1996). Tendencia que continúa en la actualidad: las explotaciones certificadas como tal ascienden a 759, ocupando una superficie de 5.035,76 hectáreas. Extensión reducida si se compara con la nacional, unas 204.238 ha. No obstante, estas cifras deben ser matizadas ya que del total citado, 249 dedican parte de su terrazgo a agricultura convencional; igualmente un $32 \%$ de las explotaciones totalmente ecológicas presenta tierras en conversión (cuadro 7). Significativo resulta, asimismo, el estudio de su localización (lámina 2): se concentran en las provin-

14 Régimen de ayudas que se tratan de favorecer la reducción del uso de fertilizantes y productos fitosanitarios en una primera etapa (superficie en reconversión con 400 ecus/ha) y en la extensificación con primas que oscilan entre 150 ecus/ha para los cultivos herbáceos y entre 400 y 600 según la variedad de leñosos acogida. 
cias de Verona y Treviso; más concretamente en el área central y especialmente en los extremos nororiental y occidental, es decir, aquélla que por sus condicionamientos fue la más apta para la intensificación en sus usos, frente a áreas como la montaña o las colinas, donde, a pesar de predominar los usos extensivos, los agricultores son reacios a un cambio en el sistema de explotación de sus tierras: prefieren intensificar o abandonar sus tierras.

Este significativo auge y su propia localización se vincula con una serie de aspectos entre los que destacan el incremento de la demanda de este tipo de productos, en un primer momento, en el mercado internacional y, posteriormente en el nacional ${ }^{15}$. Difusión esta última favorecida por la expansión experimentada por las industrias agroalimentarias que, gracias en parte a las ayudas que este reglamento prevé para la mejora de los canales de comercialización, han incorporado estos productos a los hábitos de consumo de los italianos mediante la creación de tiendas especializadas y, sobre todo, su incorporación a la oferta que presentan las grandes cadenas alimenticias. Iniciativa que trata de reducir los altos costes, la fragmentación y la escasez de productos que tradicionalmente han limitado el desarrollo de esta práctica agrícola.

En segundo lugar, la existencia de un campesinado que adopta está opción, consciente de las mayores rentas que este tipo de agricultura les proporciona frente a las prácticas tradicionales. El valor generado por la agricultura ecológica véneta ascendía a 2.443.874 euros (1997) es decir, el 1,83\% del productor interior bruto agrícola, aunque sólo era practicada en el 0,3\% de las explotaciones y afectaba al 0,5\% de la superficie agraria utilizada. La existencia de una mentalidad empresarial, como confirman las entrevistas realizadas, justifica que estas explotaciones se concentren en el área central de la región dotada de una excelente red de comunicaciones que le permiten acceder al mercado con rapidez o que sus practicantes, con una edad inferior a la media de los activos regionales, se muestren proclives a adoptar innovaciones que les permitan continuar con esta actividad productiva.

Cuadro 8

DISTRIBUCIÓN DE LOS APROVECHAMIENTOS AGRÍCOLAS (1998)

\begin{tabular}{|l|rr|rr|rr|rr|}
\hline & \multicolumn{2}{|c|}{ Cereales } & \multicolumn{2}{|c|}{ Hortalizas } & \multicolumn{2}{c|}{ Leñosos } & \multicolumn{2}{c|}{ Ganadería } \\
\cline { 2 - 10 } & \multicolumn{1}{|c|}{$\mathbf{N}^{\mathbf{0}}$ expl } & Sup & $\mathbf{N}^{\mathbf{0}}$ expl & Sup & $\mathbf{N}^{0}$ expl & Sup & $\mathbf{N}^{0}$ expl & Sup \\
\hline Veneto & 175 & 1643,07 & 89 & 469,31 & 436 & 1726,31 & 59 & 739,59 \\
\hline Belluno & 2 & 8,41 & -- & -- & 8 & 30,11 & 50 & 561,12 \\
Padova & 29 & 278,82 & 7 & 18,15 & 69 & 273,13 & --- & -- \\
Rovigo & 38 & 487,21 & 24 & 169,32 & 18 & 139,22 & --- & --- \\
Treviso & 19 & 73,82 & 20 & 64,32 & 94 & 243,13 & 4 & 67,89 \\
Venezia & 26 & 314,13 & 16 & 93,72 & 41 & 188,24 & --- & --- \\
Verona & 46 & 436,65 & 18 & 101,32 & 151 & 632,14 & 2 & 46,43 \\
Vicenza & 15 & 43,97 & 4 & 22,45 & 55 & 220,34 & 3 & 64,15 \\
\hline
\end{tabular}

Las explotaciones que practicas aprovechamientos mixtos, se han incluido en el dominante. Fuente: Ente Sviluppo Agrario del Veneto. Elaboración propia.

15 A pesar del significativo incremento de la demanda de productos ecológicos en el mercado nacional, la exportación sigue alcanzando porcentajes que se sitúan alrededor del 55\%; siendo Alemania y Francia los principales importadores. 
El análisis de los aprovechamientos dominantes reitera la concepción de esta práctica como una actividad capaz de generar rentas más elevadas que los aprovechamientos dominantes en el Véneto (cuadro 3). Frente al predominio de los cultivos herbáceos (cereales entre los que predomina el maíz y los cultivos industriales con la soja y la remolacha azucarera como aprovechamientos más significativos) en la agricultura tradicional, la no convencional se caracteriza por el dominio de los cultivos permanentes: el viñedo para vinificación y frutales ocupan el 18,9 y el 15,9\% de la superficie total, respectivamente. Aprovechamientos a los que se une la incipiente presencia de los cultivos hortícolas (84 explotaciones, $9,5 \%$ de la SAU) e incluso el olivo $(1,4 \%)$, circunscrito al microclima del lago de Garda. Esta práctica se caracteriza, consiguientemente, por una especialización muy superior a la de la agricultura convencional: ésta únicamente ocupan el 16,7\% frente al $43,7 \%$ de la biológica (cuadro 8). Debemos, por último, mencionar las actividades ganaderas localizadas preferentemente en el área montañosa y dedicadas a la cría de bovino para carne y leche.

\section{Conclusiones}

En Italia, hasta los años 80 , se consideraba que la tutela ambiental sólo podía llevarse a cabo en «países más ricos» pues numerosas empresas no podrían hacer frente a los costes adicionales que ocasionaría la adopción de tecnologías compatibles con el medio ambiente. Afirmación que se tradujo en que Italia durante muchos años se situase a la cola de los países europeos en la adaptación de las directivas comunitarias en materia ambiental y éstas, generalmente, eran aplicadas parcialmente (Franceschetti, G. y Tempesta, T., 1993). La ley Gallaso (431/85), por ejemplo, obligaba a la realización de un Piano Paesagistico Regionale antes del 31-12-86. Sin embargo, pocas regiones y, con mucho retraso, han llevado a cabo esta normativa. Afirmaciones que tratan de ser superadas con la nueva legislación regional. El Programma Regionale di Sviluppo Agricolo 1990-94, reconocía la necesidad de adoptar medidas específicas en el sector primario que permitieran conservar el medio ambiente y el paisaje especialmente en sectores colinares y montañosos. No obstante, su resultado ha sido, al igual que la aplicación de las políticas de corte ambiental emanadas de la reforma de la PAC, más bien escaso.

Esta exigua aceptación se vincula a una serie de carencias entre las que destaca en primer lugar la inexistencia de una verdadera preocupación por la cuestión ambiental. La aprobación de las denominadas medidas de acompañamiento persigue más una reducción del gasto agrario comunitario que una protección del agro europeo. Así, por ejemplo, las ayudas reguladas por estos reglamentos no cubren la pérdida de renta que ocasiona su adopción. Ante esta situación es evidente que la respuesta a estas disposiciones no ha respondido a las expectativas creadas: ningún sector económico y mucho menos el primario, donde numerosas explotaciones presentan problemas de viabilidad, estaría dispuesto a introducir unas medidas que les ocasionarán una reducción en sus ingresos.

La adopción de una verdadera política ambiental debe acompañarse, asimismo, de una simplificación en la tramitación de las ayudas. Tanto los agricultores que practican la agricultura ecológica como los que han reforestado antiguos terrazgos agrícolas se quejan de la lentitud de la administración en pagar las primas por la adopción de estas técnicas, así como de la exigüidad de éstas y la excesiva burocracia para solicitar la inclusión en superficie donde se practica la agricultura ecológica y de los altos costes iniciales que supone la adopción de técnicas compatibles con el medio ambiente. Siendo necesaria la aprobación de 
otras ayudas como una menor presión fiscal, préstamos a bajo interés, etc. que complementarían la prima que trata de compensar la pérdida de rentas con la adopción de estas técnicas.

\section{Bibliografía}

AGUILAR FERNÁNDEZ, S. (1997): El reto del medio ambiente. Conflictos e intereses en la política medioambiental europea, Alianza Universidad, Madrid.

ALBRIZIO, M. (1990): L'agricoltura ecologica in Italia, Ismea, Ministero Agricoltura, Roma.

ALTIERI, M.A. (1991): Agroecologia. Prospettive scientifiche per una nuova agricoltura, Franco Muzzio editore, Padua.

BERNARDI, R.; ZANETTO, G. y ZUNICA, (ed.) (1991): Il Veneto diversià e omogeneita di una regione: emergenze territoriali e socio-economiche, Patrón Bolonia.

BERNI, P. y FABBRIS, L. (ed.) (1996): L'agricoltura biologica nel Veneto, Consiglio Nazionale della Ricerca, Arcadia, Módena.

BOATTO, V.; DEFRANCESCO, E.; BALESTRIERI, M. y SUMAN, R. (1996): L'agricoltura ecologica nella provincia di Padova, Unipress, Padua.

BOATTO, V.; BUSTAFFA, R.; RELA, G.; SCUDELLER, A. y FAVRETTO, M.R. (1997): «Assetto produttivo dell'agricoltura biologica veneta», L'informatore agrario, 46, pp. 27-30.

DE PASQUALE, G. (1986): «Incentivi alla cooperazione in agricoltura attivate dalle misure di politica comunitarie delle strutture agrarie» en Mezzogiorno di Europa, Nápoles, pp. 437-466.

ENTI DI SVILUPPO AGRARIO DEL VENETO (1995): Atti della giornata sull' imboschimento a prioritaria funzione ambientale di terreni di pianura, Azienda Regionale delle Foreste del Veneto, Regione Veneto, Venecia.

FABIANI, G. (1986): L'agricoltura italiana tra sviluppo e crisi (1945-85), Il Mulino, Bolonia.

FRANCESCHETI, G. y TEMPESTA, T. (1987): Agricoltura e paesaggio, Arsenale, Venecia.

- (1993): La pianificazione del territorio rurale del Veneto negli anni ottanta, Unione Regionale delle Provincie del Veneto-Unipress, Venecia.

HERNÁNDEZ HERNÁNDEZ, MARÍA (1998): «Repercusiones socio-espaciales de la reforestación de tierras agrícolas en la provincia de Alicante» en IX Coloquio de Geografía Rural, Departamento de Geografía, Prehistória y Arqueología-Asociación de Geógrafos Españoles, Vitoria, pp. 269-276.

- (1999): «Los paisajes hortícolas en el Véneto: evolución y repercusiones socio-epciales», en Investigaciones Geográficas, Instituto Universitario de Geografía, Universidad de Alicante, $n^{\circ} 21$, pp. 59-80.

HUBERT, B. (1993): «Nueva política comunitaria de estructuras agrarias y de desarrollo rural y la reforma de la PAC», en Agriculturas y políticas agrarias en el sur de Europa, MAPA, Madrid, pp. 209-226.

LÁZARO ARUJO, L. y MOLINA IBÁÑEZ, M. (1986): El espacio de la CEE. La política regional, ed. Trivium, Madrid.

INEA (1995): I principali indicatori economici dell'agricoltura veneta: elaborazione di dati contabili (1990-93), Ufficio di Contabilità Agraria per il Veneto, Observatorio di Economia Agraria, Venecia. 
ISTAT (1991): Caratteristiche strutturali delle aziende agricole. $4^{o}$ Censimento generale dell'agricoltura, Provincias de Rovigo, Verona, Venecia, Treviso y Padua, Roma.

PIANO TERRITORIALE REGIONALE DI COORDINAMENTO (1993), Regione del Veneto, Edizione Canova, Venecia.

PINTON, R. (1994): «Il mercato del biologico in Veneto», Bioagricoltura $n^{\circ} 29$, Arcadia Editrice, Modena.

RUGGIERI, M. (1984): «Il recupero dei terreni abbandonati nei paesi della CEE» en Boll. Soc. Geog. Ital IX, $n^{o}$ 1, Roma, pp. 359-366.

SCARPELLI, L. (1996): Veneto, Geografia dei Sistemi Agricoli Italiani, REDA, Edizione per l'agricoltura, Roma.

SERENI, E. (1993): Storia del paesaggio italiano, Laterza, Roma.

STROPPA, M. (1992): «Dal trattato di Roma alla Riforma MacSharry: il difficile rapporto tra politica agricola e politica ambientale della CEE», Studi di economia e diritto, $n^{o} 1$, Roma.

ZANETTI, P. (1996): L'agricoltura veneta della tradizione alle sperimentazione attraverso le scuole e le stittuzione agrarie padovane, Cleup, Padua.

ZONIN, R. (1997): Aspetti economico-sociale dell'agricoltura biologica veneta, Seminario Facoltà Agraria di Ancona, Ancona. 